\title{
El impacto de los trabajos de Daniel Kahneman y colaboradores en la administración
}

\section{The impact of Daniel Kahneman and team's researches in manage- ment}

\author{
Julián Xavier Torreblanca ${ }^{1 *}$ \\ ${ }^{1}$ Facultad de Ciencias Económicas de la Universidad Adventista del Plata
}

\section{INFORMACIÓN DEL ARTÍCULO}

Historia del artículo

Recibido: 30 de julio 2016

Aceptado: 17 de octubre del 2016

\section{Palabras clave:}

Daniel Kahneman, teoría prospectiva, toma de decisiones, modelos de decisión

\section{Keywords:}

Daniel Kahneman, prospect theory, decision making, decision models

\begin{abstract}
Resumen
La toma de decisiones cumple un papel fundamental en el desempeño del administrador. Hasta las publicaciones de Daniel Kahneman, la teoría con respecto a las decisiones se abocaba a la maximización de la utilidad. El punto de vista más cercano a la realidad de la Teoría Prospectiva dio lugar a la incorporación de elementos subjetivos que influyen en el decididor. La aversión al riesgo en un escenario de ganancia y la tolerancia al mismo en situaciones de pérdida junto con la sensibilidad disminuida, se convierten en pilares para centenares de estudios que aplican la Teoría en áreas como la psicología, economía, y administración. El presente trabajo revisa la literatura pertinente a dicha aplicación en el desempeño del administrador. La categorización realizada organiza las aplicaciones en factores individuales, organizacionales y de administración pública brindando un panorama de la situación actual y lanzando propuestas de análisis futuros.
\end{abstract}

* Autor correspondiente: Julián Xavier Torreblanca. MBA, CP, LA, Doctorando en Cs. Económicas, Universidad de Buenos Aires. Becario Doctoral CONICET. Docente de la Facultad de Ciencias Económicas en la Universidad Adventista del Plata.

Dirección Postal: 25 de Mayo 99, Libertador San Martín (3103), Entre Ríos, Argentina

Correo electrónico: juliantorreblanca@doc.uap.edu.ar 


\section{Introducción}

Históricamente, las investigaciones relativas a la elección en decisiones bajo riesgo han sido estudiadas en su mayoría desde el punto de vista de la Hipótesis de la Utilidad Esperada (HUE), donde el individuo elige la mejor alternativa en base a la maximización del resultado de riqueza final (Holmes Jr., Bromiley, Devers, Holcomb y McGuire, 2011). Sin embargo, una opción más cercana a la realidad surge a partir de las propuestas de Daniel Kahneman y Amos Tversky $(1974,1979,1981,1984,1992)$. Su Teoría Prospectiva (TP) introduce elementos de la psicología para poder entender mejor por qué el individuo elige alternativas que no concuerdan con los axiomas de maximización de resultados de la HUE. La elección que realizan los individuos, según este modelo, traduce los resultados de las alternativas a valores subjetivos y luego les otorga un peso según la importancia relativa que le otorga el individuo de acuerdo a su punto de referencia. De su análisis nacen conceptos fundamentales como la sensibilidad disminuida a medida que nos alejamos del punto de referencia y la aversión a la pérdida.

Este trabajo se propone indagar, luego de presentar brevemente el modelo, la literatura que aplica la TP a facetas de la Administración, para revisar su impacto en esta última. Se analizarán las alternativas desde la perspectiva individual, colectiva y pública. Si bien es un modelo establecido a fin de la década del 70, Leavy (2010, p.2) menciona, al respecto de la TP, que: "en las últimas décadas, se ha incrementado exponencialmente el número de trabajos de investigación así como de publicaciones en las diversas áreas del conocimiento científico". Más de 7.400 artículos de investigación han citado los trabajos de Kahneman y Tversky, de los cuales 550 de ellos han sido publicados en revistas académicas de investigación en administración reconocidas internacionalmente (Holmes Jr. et al., 2011).

\section{La teoría prospectiva}

Las teorías de la decisión comenzaron a tomar relevancia con la obra seminal de Von Neumann y Morgenstern en 1947. Su libro Theory of Games and Economic Behavior intenta traer luz sobre los mecanismos que utilizan las personas al tener que decidir entre alternativas con riesgo mediante axiomas que maximizan la utilidad. La Hipótesis de la Utilidad Esperada (HUE) es desde aquel momento la teoría de decisión más desarrollada y utilizada. Para decidir, la persona utiliza una probabilidad ponderada media de los resultados que podría traer la decisión como base para evaluar las preferencias de riesgo de la persona. Esto es, la persona deriva la utilidad de la alternativa desde la posición de riqueza final (el resultado de la decisión más la riqueza actual).

En 1979, Daniel Kahneman y Amos Tversky publicaron Prospect Theory: An Analysis of Decision under Risk. En el mismo proponían un modelo descriptivo para la toma de decisiones bajo riesgo, despegándose del enfoque normativo de la HUE. Este trabajo, una crítica al modelo de la HUE donde se flexibilizan algunos de sus axiomas para explicar las inconsistencias en el comportamiento, es el mayor soporte para la Economía Conductual (en inglés Behavioral Economics). Según Moraes Ramos, Daamen y Hoogendoorn (2014), el punto de la crítica radica en que cambiando la manera en se presentan las opciones, se generan cambios predecibles en la preferencia de la decisión, situación que no debería ocurrir en el enfoque de la HUE.

La Teoría Prospectiva propone que las decisiones se basan en ganancias y pérdi- 
das medidas por un punto de referencia; por esto lo que importa, a diferencia de la HUE, no es el resultado final de ganancia o pérdida sino la ganancia o pérdida relativa a este punto. De acuerdo a Kahneman y Tversky (1979), la localización del mismo y la consecuente codificación de resultados como ganancias o pérdidas pueden ser afectadas por la formulación de los prospectos (alternativas) y por las expectativas de la persona.

La teoría de Kahneman y Tversky explica a las decisiones bajo riesgo como un proceso de dos fases. En primer lugar, la persona organiza las opciones y las reformula codificándolas por efectos para hacer más fácil la siguiente fase. La segunda fase es la evaluación. En este proceso el prospecto es valorado subjetivamente. Esta fase se descompone a su vez en dos funciones: valor (values) y ponderación de probabilidades (weights). La primera refleja el valor subjetivo del resultado, midiendo así las desviaciones desde el punto de referencia. La ponderación de probabilidades asocia un peso a cada probabilidad de ocurrencia reflejando el impacto de la misma en el prospecto. Esto tiende, por lo general, a reducir los eventos con alta probabilidad de ocurrencia y a sobreestimar los opuestos; sin embargo, también se le asigna un importancia de cero a los eventos con probabilidad extremadamente baja de ocurrir.

En los trabajos seminales de Kahneman y Tversky (1974 y 1979) se destacan efectos que distorsionan el razonamiento de la persona (heuristics y biases) y sesgan la elección. Se listan a continuación los principales efectos encontrados en sus estudios seminales y en investigaciones posteriores:

- Efecto certeza: En zona de valores positivos con respecto al punto de referencia, las personas tienden a sobrestimar el peso de resultados considerados como ciertos sobre aquellos que conllevan riesgo pero mayor retorno, por lo que escogen la opción más segura por sobre la más rentable. Según la HUE esto no debería suceder.

- Efecto reflejo: Al contrario que el anterior, al encontrarse la persona en valores negativos se tiende a tolerar más el riesgo. De esta manera, se llega a la conclusión que las personas tienen un miedo mayor a perder que a arriesgar a perder más. Los resultados inciertos tienen mayor peso que aquellos ciertos más pequeños.

- Efecto aislación o cancelación: Al elegir entre diferentes alternativas se descompone la elección para hacer más fácil la elección. Entre las alternativas surgen entonces componentes compartidos. La persona tenderá a realizar la decisión con la menor cantidad de razonamiento posible (Kahneman, 2011) por lo que los componentes compartidos serán desechados para centrarse en aquellos que distinguen a las alternativas. El problema radica en que habrá diferentes maneras de descomponer un problema generando así la posibilidad de desestimar componentes que no se apartarían si se analizara la alternativa desde otro punto de vista, con diferentes preferencias.

- Efecto segregación: Al analizar alternativas, las personas eliminan aquellos componentes que carecen de riesgo en las alternativas. La alternativa $(100,0.8 ; 200,0.2)$, por ejemplo, se codifica en dos partes: $(100,1)$ sin riesgo y $(100,0.2 ; 200$, $0.8)$ con riesgo. 
- Efecto combinación: Las personas combinarán resultados idénticos entre alternativas. Siguiendo con el ejemplo, las alternativas (100, 0.1; $100,0.1 ; 200,0.8)$ se combinan en $(100,0.2 ; 200,0.8)$.

- Efecto simplificación: La mente no puede entender el tamaño de ciertas probabilidades por lo que las redondea. $(300,0.056 ; 501,0.4)$ pasa a ser $(300,0.06 ; 500,0.4)$

- Efecto dominante: Ante una comparación rápida de alternativas, aquella que a la vista es menos favorable (dominada) es eliminada.

- Efecto porcentaje: Un incremento de $\$ 10$ en un precio total de $\$ 100$ tendrá un mayor peso que el mismo incremento en $\$ 1000$. La respuesta al estímulo es proporcional al porcentaje de cambio de dicho estímulo.

La función de valor es definida, como se estableció, por desviaciones de un punto de referencia. Es generalmente cóncava para las ganancias (positivo) y convexa para las pérdidas (negativo). La pendiente es mayor para las pérdidas que para las ganancias fundamentando el miedo a la pérdida (Figura 1).En la función de ponderación de probabilidades (weights) se presentan dos fenómenos, "disminución en la sensibilidad para probabilidad cercana a 0 (convexa) y 1 (cóncava), y por otro lado tendencia a la aversión al riesgo para las ganancias y tomador de riesgo para las pérdidas" (Lea$v y, 2010$, p.25). Esta función contribuye al patrón general de la aversión al riesgo en muchas decisiones. En la Figura 2, se observa que la mayoría de las probabilidades están infravaloradas. Se decanta, por lo tanto, la conclusión de que la suma de los valores de esta función será menor a 1 contribuyendo así a la tendencia ge- neral hacia la aversión al riesgo dado que la suma de las alternativas siempre será igual a 1 (Kahneman y Tversky, 1979).

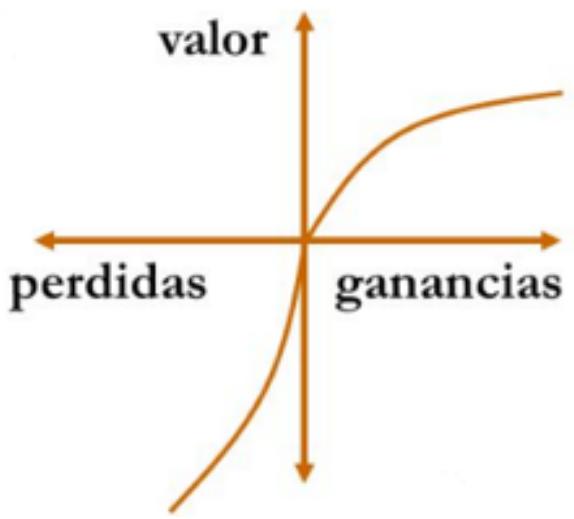

Figura 1 - Función de Valor (value)

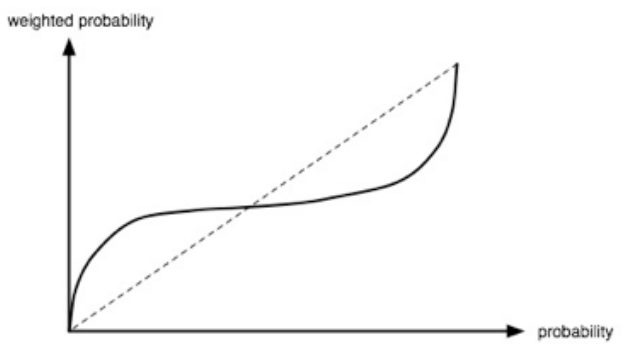

Figura 2 - Función de Ponderación de Probabilidades (weights)

A pocos años de la postulación de la TP, surgen nuevos aportes por medio de dos nuevos trabajos de Kahneman y Tversky (1981 y 1984) donde se menciona que las elecciones son influenciadas por cómo son representadas cognitivamente las perspectivas en términos de ganancias o pérdidas y sus probabilidades asociadas. Como sostiene Leavy (2010, p.18), "Al variar la descripción de las opciones uno puede influenciar sobre cómo son percibidos. Los autores de la teoría sugieren que el tomador de decisiones edita las perspectivas en formación de sus representaciones subjetivas". En la publicación Advances in prospect theory: Cumulative Representation of Uncertainty 
de Tversky y Kahneman (1992), se ajustó los trabajos anteriores para hacer posible evaluar situaciones que implicaban incertidumbre en las cuales los resultados no eran conocidos. En esta nueva versión el modelo se expande a varios nuevos aspectos y suma el emplear acumulaciones en lugar de decisiones separadas en el momento de la ponderación del peso. A la adecuación se la conoce como Teoría Prospectiva Acumulada (TPA). Las herramienta de recolección de datos de Kahneman y Tversky fue aplicada positivamente por de Sousa Barros y dos Santos Felipe (2015) en Brasil, demostrando que los conceptos siguen vigentes y son aplicables a nuestro entorno.

Impacto y aplicaciones de la teoría prospectiva en la administración

\section{Nivel individual}

La TP es un modelo de toma de decisiones bajo riesgo a nivel individual. Las áreas más favorables de su aplicación, dadas estas características, son las finanzas y los seguros donde las actitudes frente al riesgo asumen un papel central. El área más destacada e investigada es la financiera donde los trabajos han explicado mediante la TP, entre otras, por qué algunos activos tienen retornos medios más altos que otros, por qué algunos individuos compran y otros venden acciones en el mismo momento, cómo debería estar compuesto el portafolio de una persona dada su aversión al riesgo y su estado actual (Barberis, 2013).

El área de los seguros es otro sector donde las actitudes al riesgo juegan un papel central. Aquí es más que importante destacar a la TP en cada estrategia del administrador. Los mercados más importantes de seguros personales de acuerdo a Barberis (2013) son los de propiedad, vida y salud. Todos han sido investigados desde la perspectiva de la TP. La base de la TP para los seguros es que el individuo adquiere un seguro desde un marco de ganancia bajo el punto de referencia de la sanidad ante la probabilidad de que ésta cambie. Marquis y Holmer (1996) afirmaron que el valor de la prima no influye en la decisión hasta que sobrepasa el valor de gasto irrelevante del individuo. Ahondando en el tema, Schneider (2004) estudió a individuos con bajos recursos que rompían con esta regla al no tener seguro de salud. Encontró un cambio en el punto de referencia de la salud al dinero donde el individuo se encontraba a sí mismo en una situación de pérdida y toleraba el riesgo. Siguiendo lo dicho por Kahneman y Tversky en 1979, estos individuos solo se asegurarán ante el hecho certero de que la pérdida ocurrirá. Utilizando la TPA Schneider (2004) estableció que el individuo se asegura porque otorga una sobre importancia (overweight) a la pequeña posibilidad de enfermarse. Sin embargo, los individuos de bajos recursos otorgan una importancia inferior (underweight) a la posibilidad dado que su punto de referencia más fuerte es el ingreso diario y generalmente están en situaciones de restricción de liquidez, por lo que no se aseguran.

Holmes Jr. et al. (2011) destacan que las conclusiones de la TP relativas a la aversión al riesgo pueden ser utilizadas al evaluar decisiones de compensación a los ejecutivos de una organización. El plan de compensación, y más específicamente el precio de ejercicio de su opción de acciones comparado con el precio real de la acción, se convertirá en su principal punto de referencia. Una acción en alza podría hacer que el ejecutivo adopte un marco de ganancia haciéndolo tener actitudes de aversión al riesgo. A la vez, altos incentivos por metas cumplidas pueden hacer que adopte un marco de pérdida y tolerancia al riesgo. Por lo tanto, a dife- 
rencia de la teoría existente al momento del estudio, la compensación puede motivar tanto la aversión como la tolerancia al riesgo dependiendo el marco de ganancia o pérdida que los ejecutivos den a las consecuencias de sus decisiones.

Las investigaciones que han utilizado a la TP para analizar toma de decisiones bajo riesgo en negociaciones han destacado tres resultados. Primero, las negociaciones en marcos de pérdida pueden tornarse tolerantes al riesgo con comportamientos agresivos. En el lado opuesto, negociaciones en marcos de ganancias pueden ser adversas al riesgo y mostrar comportamientos cooperativos (Holmes Jr. et al., 2011). La investigación de Neale y Bazerman (1985) estudió comportamientos de negociación concluyendo que los negociadores enmarcados en pérdidas actuaban más competitivamente haciendo menos concesiones que los que lo se enmarcaban en un comportamiento de ganancias. La segunda consecuencia decanta de la primera, los negociadores en marco de pérdida dificultarán el alcance de un acuerdo. En tercer lugar, Northcraft, Brodt y Neale (1995, citado en Holmes Jr. et al., 2011) propusieron que debido a la curvatura de la función de valor los resultados cerca del punto de referencia del negociador tendrán mayor valor por lo que una concesión de la oposición en este aspecto sería valorada con un plus subjetivo.

La mayoría de los estudios que utilizaron TP para medir motivación lo hicieron sobre la función de valor, especialmente sobre el concepto de aversión al riesgo y en menor medida puntos de referencia y sensibilidad disminuida. Los estudios de Steel y Konig (2006) encontraron que, en consistencia con la aversión al riesgo, los individuos estaban más motivados a realizar tareas cuando éstas ofrecían ganancia inmediatas y pérdidas retrasadas. El estudio de Crawford y Meng (2011) realizado a los conductores de taxi de Nueva York, encontró que el número de horas trabajadas en un día es inversamente proporcional al sueldo horario promedio. Utilizando TP, el conductor deriva su resultado del día midiendo la diferencia entre su resultado diario y un marco de referencia propuesto de antemano. En particular, debido al miedo a la pérdida, ganar $\$ 100$ menos no es igual a ganar $\$ 100$ más. Por esta razón una vez alcanzado el objetivo dejará de trabajar, de otra manera seguirá trabajando hasta que su punto de referencia cambie a horas trabajadas y el ingreso por hora extra tenga una ponderación menor al costo de esa hora.

En el ámbito de la administración de recursos humanos, Highhouse y Johnson (1996) argumentaron que el proceso de selección incluye una decisión de riesgo con resultados positivos y negativos. De acuerdo a los estudios, el punto de referencia de los administradores se ubica en el desempeño de los candidatos con respecto a las incumbencias del trabajo. Desde la perspectiva de la aversión al riesgo, los autores encontraron que se elegían a individuos cuyo desempeño no representaba un cambio significativo al anterior trabajador. De acuerdo a otro factor de la TP, la sensibilidad disminuida, la diferencia entre candidatos será percibida con mayor valor cuando se trate de números de menor magnitud (errores 5\% contra 6\%) que cuando sea una de mayor magnitud (no errores 95\% contra 94\%). Esto es consistente con la figura cóncava de la función de valor. Variando la presentación de la información, los administradores encontrarán mayores diferencias o similitudes entre los candidatos (Wong and Kwong, 2005a en Holmes Jr. et al. 2011).

La TP es aplicable también a decisiones en el área de la salud. Especialmente en el sector de medicina preventiva, los 
individuos pueden variar la decisión de realizarse un test o evitarlo dependiendo de su marco de referencia. Un test rutinario que puede llegar a detectar cáncer de piel envía un mensaje de pérdida respecto al marco de referencia de la persona. Siguiendo a la TP, en esta situación el individuo optará por tomar el riesgo antes que conocer la noticia. Por otro lado utilizar bloqueador solar para prevenir cáncer de piel, el mismo fin visto desde otra perspectiva, podría ser interpretado como una situación de ganancia por el individuo logrando prevenir la enfermedad (Schwartz, Goldberg y Hazen, 2008).

A pesar de que la TP fue creada originalmente como una teoría de decisión bajo riesgo, Thaler (1980) argumentó que la teoría también podía ser útil para entender elecciones libres de riesgo. Los trabajos de Knetsch y Thaler (1989 y 1980, citados por Barberis, 2013), aportan estudios sobre el fenómeno del apego junto al miedo a la pérdida propuesto por la TP. La conclusión es que el miedo a la pérdida de un objeto que el individuo poseyó por un tiempo hace que no lo quiera intercambiar por otro similar. Inclusive al monetarizar la oferta para el intercambio, el precio requerido por el individuo era significativamente mayor al normal. El administrador a cargo de la comercialización puede ver en esto una ventaja simple al establecer una rebaja en determinado producto. El apego hará que en un futuro cuando el individuo vuelva a requerir el producto y se encuentre con el precio más alto el miedo a la pérdida de irse sin el ítem hará que lo compre a pesar del precio. Barberis (2013) confirma esto al sugerir que intercalando precios altos y bajos el administrador puede inducir al individuo a pagar un precio promedio por sobre el valor real del producto. De un modo similar, el individuo codificará un precio más bajo al de referencia como una ganancia y uno más alto como una pérdida por lo que esto también disparará la consecuente aversión o tolerancia al riesgo de comprar el producto. Greenleaf (1995) advierte que ofrecer descuentos de manera regular puede hacer que el individuo adopte este precio como referencia, por lo que aconseja utilizar promociones irregulares para evadir esta situación. La TP puede también ser incluida en las estrategias de publicidad y posicionamiento. Precio, calidad e imagen general son las dimensiones más importantes del punto de referencia del individuo. El estudio de Biswas y Blair (1991) demuestra que la publicidad puede modificar el punto de referencia de un producto (comúnmente, el precio). Al promocionar el producto como de calidad mayor a la normal o diferencias con la competencia el individuo cambiará su punto de referencia. Sin embargo, el no lograr satisfacer esas expectativas pondrá al producto en zona de pérdida con el consecuente aumento de la tolerancia al riesgo (probar otro producto). El posicionamiento es vital para generar el primer marco de referencia en el individuo; cambiarlo resulta mucho más difícil y oneroso.

En 2009, Koszegi y Rabin propusieron una manera de incorporar la TP a un modelo dinámico de elección de consumo. A través del miedo a la pérdida un individuo será más sensitivo a la información de que su nivel de consumo en algún punto será más bajo que lo esperado que a la novedad de que será más alto. Esto causa el ahorro por precaución, un individuo ahorrará más ahora para evitar el dolor de encontrarse más adelante ante una disminución forzada del nivel de consumo que había planeado. Por otro lado, un incremento en el ingreso de la persona genera desajustes en la importancia y los pesos que el individuo coloca sobre los prospectos. Brunnermeier (2004) estudió el impacto de ganar la lotería en el consumo desde tres pilares de la TP: preferencias de riesgo 
en zonas de ganancia y pérdida, miedo a la pérdida y sensibilidad disminuida. Las conclusiones llevaron a observar errores de juicio al asignar el peso a cada opción durante los primeros meses debido a la aceptación de haber ganado la lotería y encontrarse en otro nivel de ingreso. Luego de un tiempo el individuo utilizaba los efectos de la TP para simplificar el evento y ajustar su consumo.

\section{Nivel organizacional}

Ciertos trabajos han extrapolado la aversión al riesgo propuesta por la TP a un nivel organizacional general para estudiar el comportamiento frente al riesgo de la organización como un todo. Wood (2009, citado en Holmes Jr. et al., 2011) concluyó, a través de un estudio de adquisiciones, que un declive en la demanda de la industria puede crear un marco de pérdida que facilite comportamientos de tolerancia al riesgo. En el mismo artículo se menciona un trabajo de Abrahamson y Rosekpof (1993) donde se encontró que muchas organizaciones copian las innovaciones de sus competidores por un comportamiento de aversión al riesgo, dado que perciben que el valor de la amenaza a quedar desactualizados es mucho más importante que la de tener una ventaja competitiva propia.

Shimizu (2007) estudió los factores en los cuales la TP puede extenderse desde un nivel individual a un organizacional cuando se debe decidir sobre la escisión de una compañía previamente adquirida. Los resultados mostraron que aunque un análisis comprensivo de toda la situación sería ideal, las organizaciones tendían a basarse en experiencias pasadas gracias a los efectos de aislación y simplificación. Con estos efectos, se podrían llegar a paralizar los esfuerzos individuales y organizacionales para obtener una solución innovativa, especialmente en organizaciones con altas pérdidas. Si bien las decisiones en una organización debían ser puramente racionales, las tendencias y comportamientos individuales y organizacionales dominaban las decisiones. Si los administradores entendieran estas preferencias, podrían ser proactivos para disminuir su impacto.

Otro punto de estudio ha sido la aversión al riesgo en las interacciones entre las organizaciones y los grupos de interés externos. Siguiendo a la TP donde un marco de pérdida fomenta el riesgo, los acreedores adoptan este modelo y no realizan sus pérdidas forzando a los deudores a tomar medidas drásticas. Al mismo tiempo, y por el mismo fenómeno, los deudores entran en un comportamiento de tolerancia al riesgo tratando de hacer lo necesario para atender las demandas de aquellos grupos de interés críticos para la supervivencia inmediata, generalmente a expensas de otro grupo (Holmes et al., 2011). Las decisiones en salud y la TP también pueden desarrollarse a nivel organizacional. En la década de 1980 se crearon la mayoría de las evaluaciones económicas de la salud pública, siendo en ese momento la HUE la teoría de decisión por excelencia. Desde ese entonces, se han encontrado tendencias que llevan a pensar en que la TP ofrece un mejor marco que la HUE (Abellan-Perpiñan, Bleichrodtb y Pinto, 2009). Su estudio exploró esta idea y fundamentó que la TP es mejor al evaluar las preferencias en las decisiones de riesgo. La TP fue mucho más consistente con las elecciones realizadas. Utilizar el enfoque de la TP, tanto de manera individual como organizacional, llevará a que las políticas públicas de salud se enfoquen en promover un comportamiento preventivo en el individuo.

\section{Nivel de administración pública}

La TP ha sido aplicada en estudios en áreas externas de la administración públi- 
ca, como ser: disuasión, crisis de estabilidad, negociación pública, acción militar y disputas diplomáticas (Bromiley y Rau, 2010). En el ámbito interno también es aplicable a decisiones sobre presupuesto, reputación, credibilidad, y recursos humanos. La mayoría de los trabajos enfatizan la diferencia en el comportamiento del administrador cuando negocia desde el marco de ganancia y el de pérdida. Weyland (2002) introduce un ejemplo con líderes de democracias frágiles latinoamericanas de los '90 que al asumir el poder se encontraron en una zona de pérdida por un ámbito de severa crisis económica. El trabajo se extiende sobre el impacto de la hiperinflación como marco de referencia y cómo esto fomentó drásticas medidas neoliberales en estos países (Menem en Argentina, Fujimori en Perú y Collor en Brasil). Sin embargo, ellas causaron poco revuelo y fueron aceptadas favorablemente por el público. El gobierno de Pérez en Venezuela, pese a estar en una situación similar, luchó con grandes protestas y revueltas. De esta situación Weyland resalta dos puntos de la TP. En primer lugar, la crisis hiperinflacionaria posicionó a los nuevos gobiernos en zonas de pérdida e hizo que tuvieran comportamientos tolerantes al riesgo. Para explicar lo sucedido en Venezuela llega al segundo punto, el marco de referencia del público. En los tres primeros países la situación era conocida y por lo tanto la tolerancia al riesgo del pueblo también lo era. En Venezuela, la inflación real era de conocimiento más limitado por el público ya que el gobierno saliente había tratado de ocultar su divulgación, haciendo cambiar el marco de referencia con el que el público evaluó las medidas de Pérez, causando malestar y alzamientos.

Explicar las decisiones de administración pública desde la óptica de la TP resulta mucho más sencillo que hacerlo desde la HUE. Elms (2004, citado en Vis, 2009) se expande sobre políticas de bienestar y diferencias presupuestarias en esta área en países con situaciones económicas similares. Un caso similar es el de la intervención militar o diplomática en conflictos en territorios lejanos con poca injerencia en la economía local. Levy (1992) observa grandes beneficios en la utilización de la TP en el estudio de las relaciones internacionales, negociación y mediación. Usando los preceptos de marco de referencia y presentación de las alternativas, el autor explica por qué la Strategic Arms Limitations and Reductions Act entre Estados Unidos y Rusia demandó tanto tiempo y lo opuesto para las negociaciones por el intercambio de oleaginosas. El primero fue enmarcado negativamente dado que ambas potencias disminuían su poder bélico. El segundo, con elementos más simples, tuvo un marco de negociación positivo donde el consenso era lo primero que se buscaba.

La cultura y la voz (representación) son otros dos aspectos en los que se ha investigado la aplicación de la TP en el sector público, tanto desde el sector de la toma de decisiones como el de la justicia organizacional. El estudio de Paddock et al. (2014) relacionó la existencia de una función de valor para la voz congruente con la TP en nueve países con diferentes culturas. Los resultados muestran que la función de valor puede ser generalizada en el acceso a la voz, pero no así para el nivel de acceso a la voz donde el punto de referencia varió significativamente entre las culturas. En la misma línea, Hunton, Hall y Price (1998, citados por Holmes Jr. et al., 2011) agregan que la voz influencia positivamente los puntos de vista de los individuos acerca de la justicia y decisión en la organización. Además, en congruencia con la sensibilidad disminuida, contra más alto es el nivel jerárquico menos ponderación tendrá un aumento de dicha voz. 


\section{Conclusiones}

El aporte sustancial de la TP a la administración se da en el área del estudio de la toma de decisiones, tarea central en el desempeño del administrador. Su impacto en el trabajo del administrador se destaca en proponer una visión diferente, descriptiva, del juicio, la elección y la decisión entendiendo que las mismas serán sesgadas por aquello que diferencia al humano de una máquina. Este modelo teórico de economía conductual se aleja del enfoque clásico normativo y refleja con mayor certeza la realidad de una decisión. El administrador no decide en un mundo de laboratorio donde las variables están definidas y los resultados siguen una lógica sino que debe hacer frente a altos niveles de incertidumbre con escenarios ambiguos, complejos, únicos, dependientes de variables desconocidas e imprevisibles, por lo tanto un modelo descriptivo se impone sobre uno normativo. A su vez, la Teoría puede ser utilizada de manera diaria por el administrador para entender las variables y sesgos personales que interactúan en sus decisiones y reconocer aquellos puntos de referencia con los que mide las decisiones y posibles resultados.

El administrador siempre buscará lograr el mejor desempeño de aquello sobre lo que administra. Al conocer la propuesta de Kahneman entiende que los prospectos serán afectados por aquellos efectos de los métodos heurísticos utilizados por él para facilitar el proceso de evaluación y que sus decisiones tendrán un marco y un punto de referencia únicos que harán variar su predisposición al riesgo. Reconocerá también aquellos sesgos y desviaciones personales que podrían afectar la decisión y tratará de bloquearlos. Tres puntos importantes en este sentido que el administrador extraerá de los trabajos de Kahneman son que el valor económico no será tan importante como la valoración subjetiva del cambio económico, que su sensibilidad irá disminuyendo a medida que el resultado se aleje del punto de referencia y que el orgullo tiene un papel mucho más importante que lo que piensa a la hora de reconocer una pérdida o error.

Al ser un modelo descriptivo, Kahneman no propone soluciones a estos sesgos más que reconocerlos y tratar de evitarlos a través de casos similares. Un pensamiento clave de la TP, que se diferencia de las teorías anteriores a Kahneman, es que el individuo necesita ayuda para tomar decisiones sin sesgos. Las heurísticas que influencian el pensamiento desvían la elección a puntos irracionales y contra productivos. Si bien es imposible eliminarlos, el conocimiento de que existen deberá ser suficiente para que el administrador los reconozca, compare y bloquee, pudiendo así decidir de manera racional sin ilusiones cognitivas.

Analizando cada decisión desde la perspectiva de Kahneman, el administrador podrá también reconocer los patrones en el comportamiento de sus administrados, informarlos y guiarlos de la manera adecuada para lograr aunar los puntos de referencia. Entenderá que ellos también tendrán sus propios preconceptos y sesgos afectando la manera en que analizan el prospecto y la decisión tomada. La manera en presentar la información tendrá un gran impacto sobre la reacción que reciba. Entendiendo a Kahneman estudiará la tolerancia o aversión al riesgo necesaria y preparará la presentación del prospecto de acuerdo a lo que se necesite.

Si bien el modelo ha sido investigado durante los últimos años, su alcance y aplicaciones en la administración dejan ver vastos espacios donde todavía es ne- 
cesario ahondar en el conocimiento. En sentido teórico todavía se necesita de investigaciones que utilicen la TP de una manera homogénea sin dividir sus com- ponentes. Se necesita, por último, de estudios que sustenten de un modo práctico la teoría generada, especialmente en lo referido a decisiones colectivas. 


\section{Referencias}

Abellan-Perpiñan, J., Bleichrodtb, H., y Pinto, J. (2009). The Predictive Validity of Prospect Theory versus Expected Utility in Health Utility Measurement. Journal of Health Economics, 28, 1039-1047.

Bamberger, P., y Fiegenbaum, A. (1996). The Role of Strategic Reference Points in Explaining the Nature and Consequences of Human Resource Strategy. Academy of Management Review, 21, 926-958.

Barberis, N. (2013). Thirty Years of Prospect Theory in Economics: A Review and Assessment. Journal of Economic Perspectives, 27, 1, 173-196.

Biswas, A. y Blair, E. (1991). Contextual Effects of Reference Prices in Retail Advertisements. Journal of Marketing, 55, 3, 1-12.

Bowman, E. (1982). Risk Seeking by Troubled Firms. Sloan Management Review, 23, 33-42.

Bromiley, P., y Rau, D. (2010). Risk Taking and Strategic Decision Making. En P. Nutt y D. Wilson (Eds.) Handbook of Decision Making. Chichester, West Sussex, U.K.: John Wiley.

Brunnermeier, M. (2004). Learning to Reoptimize Consumption at New Income Levels: A Rationale for Prospect Theory. Journal of the European Economic Association, 2, 1, 98-114.

Crawford, V., y Meng, J. (2011). New York City Cab Drivers' Labor Supply Revisited: Reference-Dependent Preferences with Rational-Expectations Targets for Hours and Income. American Economic Review, 101, 5, 1912-1932.

de Sousa Barros, T. y dos Santos Felipe, I. (2015). Teoria do Prospecto: Evidên- cias Aplicadas em Finanças Comportamentais, Revista de Administração FACES Journal, 14, 4, 75-95.

Devers, C., Wiseman, R. y Holmes, R. (2007). The Effects of Endowment and Loss Aversion in Managerial Stock Option Valuation. Academy of Management Journal, 50, 191-208.

Greenleaf, E. (1995). The Impact of Reference Price Effects on the Profitability of Price Promotions. Marketing Science, 14, 1, 82-104.

Highhouse, S. y Johnson, M. (1996). Gain/ Loss Asymmetry and Riskless Choice: Loss Aversion in Choices Among Job Finalists. Organizational Behavior and Human Decision Processes, 68, 225-233.

Holmes Jr., R., Bromiley, P., Devers, C., Holcomb, T. y McGuire, J. (2011). Management Theory Applications of Prospect Theory: Accomplishments, Challenges, and Opportunities. Journal of Management, 37, 4, 1069-1107.

Kahneman, D. y Tversky, A. (1979). Prospect Theory: An Analysis of Decision under Risk. Econometrica, 47, 2, 263-292.

Kahneman, D. y Tversky, A. (1984). Choices, Values, and Frames. American Psychologist, 39, 4, 341-350.

Kahneman, D. (2011). Thinking, Fast and Slow. New York: Farrar, Straus and Giroux.

Koszegi, B. y Rabin, M. (2009). Reference-Dependent Consumption Plans. American Economic Review, 99, 3, 909-936.

Leavy, S. (2010). Teoría de las Decisiones: Teoría Prospectiva. Anales de la XLV Reunión Anual de la Asociación Argentina de Economía Política, 1-30.

Levy, J. (1992). Prospect Theory and International Relations: Theoretical 
Applications and Analytical Problems. Political Psychology, 13, 283310.

Marquis, S. y Holmer, M. (1996). Alternative Models of Choice under Uncertainty and Demand for Health Insurance. The Review of Economics and Statistics, 78, 3, 421-427.

Milburn, T. y Isaac, P. (1995). Prospect Theory: Implications for International Mediation. Peace and Conflict: Journal of Peace Psychology, 1, 4 , 333-342.

Moraes Ramos, G., Daamen, W. y Hoogendoorn, S. (2014). A State-of-theArt Review: Developments in Utility Theory, Prospect Theory and Regret Theory to Investigate Travellers' Behaviour in Situations Involving Travel Time Uncertainty. Transport Reviews, 34, 1, 46-67.

Neale, M. y Bazerman, M. (1985). The Effects of Framing and Negotiator Overconfidence on Bargainer Behavior. Academy of Management Journal, 28, 34-49.

Paddock, E., Ko, J., Cropanzano, R., Bagger, J., El Akremi, A., Camerman, J., et al. (2014). Voice and Culture: A Prospect Theory Approach. Journal of Behavioral Decision Making, 28, 167-175.

Schneider, P. (2004). Why should the poor insure? Theories of Decision-Making in the Context of Health Insurance. Health Policy and Planning, 19, 6, 349-355.

Schwartz, A., Goldberg, J. y Hazen, G. (2008). Prospect Theory, Reference Points, and Health Decisions. Judgment and Decision Making, 3, 2, 174-180.

Shimizu, K. (2007). Prospect Theory, Behavioral Theory, and the Threat-Rigidity Thesis: Combinative Effects on Organizational Decisions to Di- vest Formerly Acquired Units. Academy of Management Journal, 50, 6, 1495-1514.

Steel, P., y Konig, C. (2006). Integrating theories of motivation. Academy of Management Review, 31, 889-913.

Thaler, R. (1980). Toward a Positive Theory of Consumer Choice. Journal of Economic Behavior and Organization, 1, 1, 39-60.

Tversky, A., y Kahneman, D. (1974). Judgment under Uncertainty: Heuristics and Biases, Science, 185, 4157, 1124-1131.

Tversky, A., y Kahneman, D. (1981). The Framing of Decisions and the Psychology of Choice. Science, 211, 4481, 453-458.

Tversky, A., y Kahneman, D. (1992). Advances in Prospect Theory: Cumulative Representation of Uncertainty. Journal of Risk and Uncertainty, 5, 4, 297-323.

Van Buiten, M., y Keren, G. (2009). Speaker-listener incompatibility: Joint and separate processing in risky choice framing. Organizational Behavior and Human Decision Processes, 108, 106-115.

Vis, B. (2009). Studying Political Decision-Making Using Prospect Theory. Amsterdam: VU University. Recuperado de https://www.researchgate. net/publication/228435173_Studying_Political_Decision-Making_ Using_Prospect_Theory

Von Neumann, J., y Morgenstern, O. (1947). Theory of games and economic behavior. Princeton: Princeton University Press.

Weyland, K. (2002). The Politics of Market Reform in Fragile Democracies: Argentina, Brazil, Peru, and Venezuela. Princeton and New York: Princeton University Press. 
